\title{
Coincident primary breast lymphoma and gastrointestinal stromal tumor: case series and molecular mechanisms
}

This article was published in the following Dove Press journal:

OncoTargets and Therapy

\author{
Ethan B Ludmir ${ }^{1, *}$ \\ Tyler Gutschenritter ${ }^{1, *}$ \\ Chelsea C Pinnix' \\ Jillian R Gunther' \\ Loretta J Nastoupil ${ }^{2}$ \\ Joseph D Khoury ${ }^{3}$ \\ $\mathrm{L}$ Jeffrey Medeiros ${ }^{3}$ \\ Bouthaina S Dabaja' \\ Sarah A Milgrom' \\ 'Department of Radiation Oncology, \\ The University of Texas MD Anderson \\ Cancer Center, Houston, TX, USA; \\ ${ }^{2}$ Department of Lymphoma/Myeloma, \\ The University of Texas MD Anderson \\ Cancer Center, Houston, TX, USA; \\ ${ }^{3}$ Department of Hematopathology, \\ The University of Texas MD Anderson \\ Cancer Center, Houston, TX, USA \\ *These authors contributed equally \\ to this work
}

Correspondence: Sarah A Milgrom Department of Radiation Oncology, MD Anderson Cancer Center,

1515 Holcombe Boulevard, Unit 97,

Houston, TX 77030, USA

Tel +l 7135632345

Fax +I 7135632366

Email samilgrom@mdanderson.org

\begin{abstract}
Gastrointestinal stromal tumor (GIST) is an uncommon mesenchymal tumor, and has been shown to be associated with synchronous or metachronous second malignancies. Rare cases of coincident GIST and non-Hodgkin lymphomas (NHL) have been reported previously. Here, we report two cases of GIST and coincident primary breast lymphoma, an uncommon subtype of extranodal NHL. We propose that the exceedingly low likelihood of both these cancers occurring in these two patients by chance warrants examination for possible common oncogenic pathways in these lesions, possibly involving shared anti-apoptotic mechanisms. Further research is vital to elucidate common oncogenic pathways between such rare lesions. Keywords: diffuse large B-cell lymphoma, mucosa-associated lymphoid tissue lymphoma, anti-apoptosis, gastrointestinal stromal tumor, primary breast lymphoma
\end{abstract}

\section{Introduction}

Gastrointestinal stromal tumor (GIST) is a rare mesenchymal tumor with an estimated incidence of 14.5 per million; 5,000 new GIST cases are diagnosed annually in the USA. ${ }^{1}$ Most cases of GIST are sporadic, with only $5 \%$ being attributable to genetic syndromes. ${ }^{1}$

The diagnosis of synchronous or metachronous malignancies in patients with GIST has been reported to occur in $17 \%-33 \%$ of cases. ${ }^{2-4}$ The most common coincident malignancies are carcinomas of the prostate gland, kidney, and bladder. ${ }^{2}$ Less commonly, non-Hodgkin lymphoma (NHL) has been shown to have significantly increased occurrence in patients with GIST, both before and after diagnosis of GIST. ${ }^{2}$ The literature suggests that gastrointestinal (GI) tract extranodal NHL (ie, - mucosaassociated lymphoid tissue [MALT] lymphoma) is the most common NHL subtype observed in patients with coincident GIST and NHL. ${ }^{5,6}$

To our knowledge, no case report exists describing GIST with synchronous or metachronous primary breast lymphoma (PBL). PBL is an exceedingly rare subtype of extranodal NHL, with an incidence of approximately three per million in the USA. ${ }^{7}$ Diffuse large B cell lymphoma (DLBCL) is the most common histology of PBL with extranodal marginal zone (MALT) lymphoma being the next most common. ${ }^{7}$ The literature for synchronous cancers in PBL is much scarcer than that for GIST, with coincident invasive ductal carcinomas of the breast being the most common type of synchronous tumor with PBL. ${ }^{6,8} \mathrm{Here}$, we report two cases of coincident PBL and GIST. We discuss the pathogenesis of each neoplasm and the existing literature addressing 
possible common pathways, potentially through anti-apoptotic mechanisms, involved by both that could explain this clinical observation.

\section{Case series}

\section{Case I}

A 76-year-old woman presented after an abnormality was detected in her left breast on screening mammography. Core biopsy revealed the lesion to be DLBCL, positive for Bcl-2 (diffuse), CD20, and CD45 positive. Subsequent fluorodeoxyglucose positron emission tomography (PET) and CT scans during staging evaluation for her PBL revealed a $5.0 \times 6.0 \mathrm{~cm}$ mass involving the duodenum and the head of the pancreas (Figure 1). Biopsy of the duodenal/pancreatic mass revealed GIST, staining positive for CD117 and CD34. The patient's family history was notable for first-degree relatives with breast, lung, and colon cancers, and the patient's social history was notable for remote tobacco usage (less than five pack-years) and chronic moderate alcohol consumption (two alcoholic servings daily).
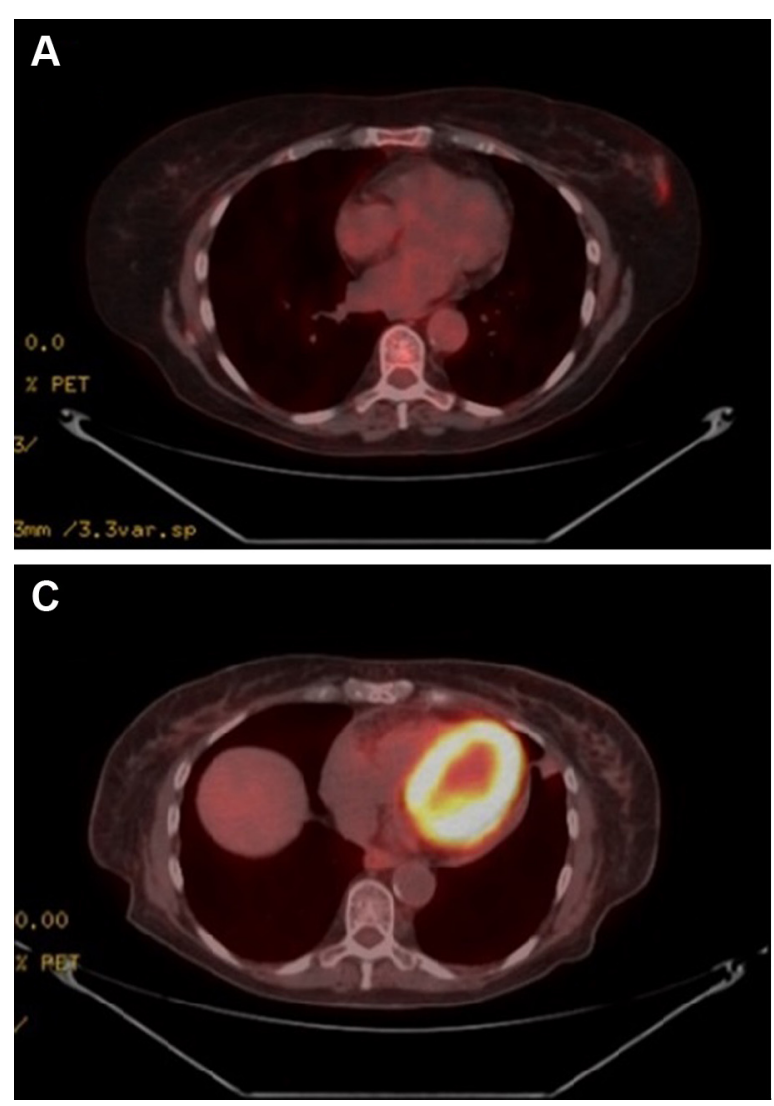
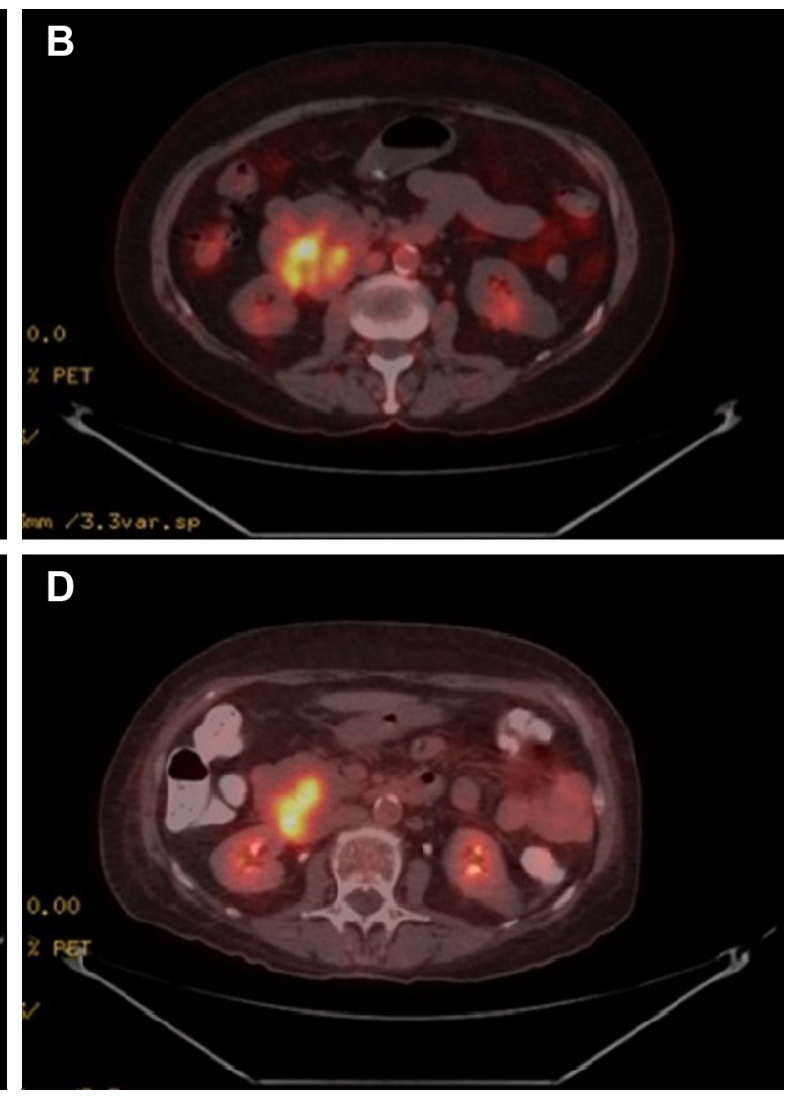

The patient underwent systemic therapy with six cycles R-CHOP (rituximab/cyclophosphamide/doxorubicin/ vincristine/prednisolone) for DLBCL with concurrent imatinib mesylate for GIST. R-CHOP cycles were delivered every 21 days, with dosing as follows: rituximab intravenous (IV) $375 \mathrm{mg} / \mathrm{m}^{2}$, cyclophosphamide IV $750 \mathrm{mg} / \mathrm{m}^{2}$, doxorubicin IV $50 \mathrm{mg} / \mathrm{m}^{2}$, and vincristine IV $1.4 \mathrm{mg} / \mathrm{m}^{2}$ all on day 1 , with prednisone per oral (PO) $100 \mathrm{mg} / \mathrm{m}^{2}$ daily on days 1-5. Concurrent with R-CHOP cycles, $400 \mathrm{mg}$ daily PO imatinib mesylate was given. No unexpected increased toxicities were observed with concurrent administration of R-CHOP and imatinib. After 10 months of imatinib (5 months after R-CHOP completion, continued at $400 \mathrm{mg}$ daily PO dosing), the patient had repeat PET/CT performed to pre-operatively assess the duodenal GIST; this PET/CT revealed resolution of the breast mass, with interval decrease in size $(4.6 \times 5.2 \mathrm{~cm})$ and central cavitation of the duodenal/ pancreatic mass suggesting treatment-related necrosis, with continued metabolic activity suggesting persistent disease despite treatment response (Figure 1). Patient, therefore,

Figure I Imaging for Case I.

Notes: Pre-treatment (A, B) and post-treatment (C, D) fused axial PET/CT images of the patient's left-sided primary breast lymphoma (A, C) and gastrointestinal stromal tumor of the pancreatic head (B, D). Pre-treatment imaging revealed mildly FDG-avid left breast changes at the site of biopsy-confirmed DLBCL (A), with resolution of these findings after six cycles of R-CHOP chemotherapy (C). Pre-treatment imaging revealed a $5.0 \times 6.0 \mathrm{~cm}$ duodenal/pancreatic head mass with significant FDG avidity (B); this decreased in size to $4.6 \times 5.2 \mathrm{~cm}$ after 10 months of imatinib, with continued FDG avidity, suggesting persistent disease despite partial response (D).

Abbreviations: DLBCL, diffuse large B cell lymphoma; FDG, fluorodeoxyglucose; PET, positron emission tomography; R-CHOP, rituximab/cyclophosphamide/doxorubicin/ vincristine/prednisolone. 

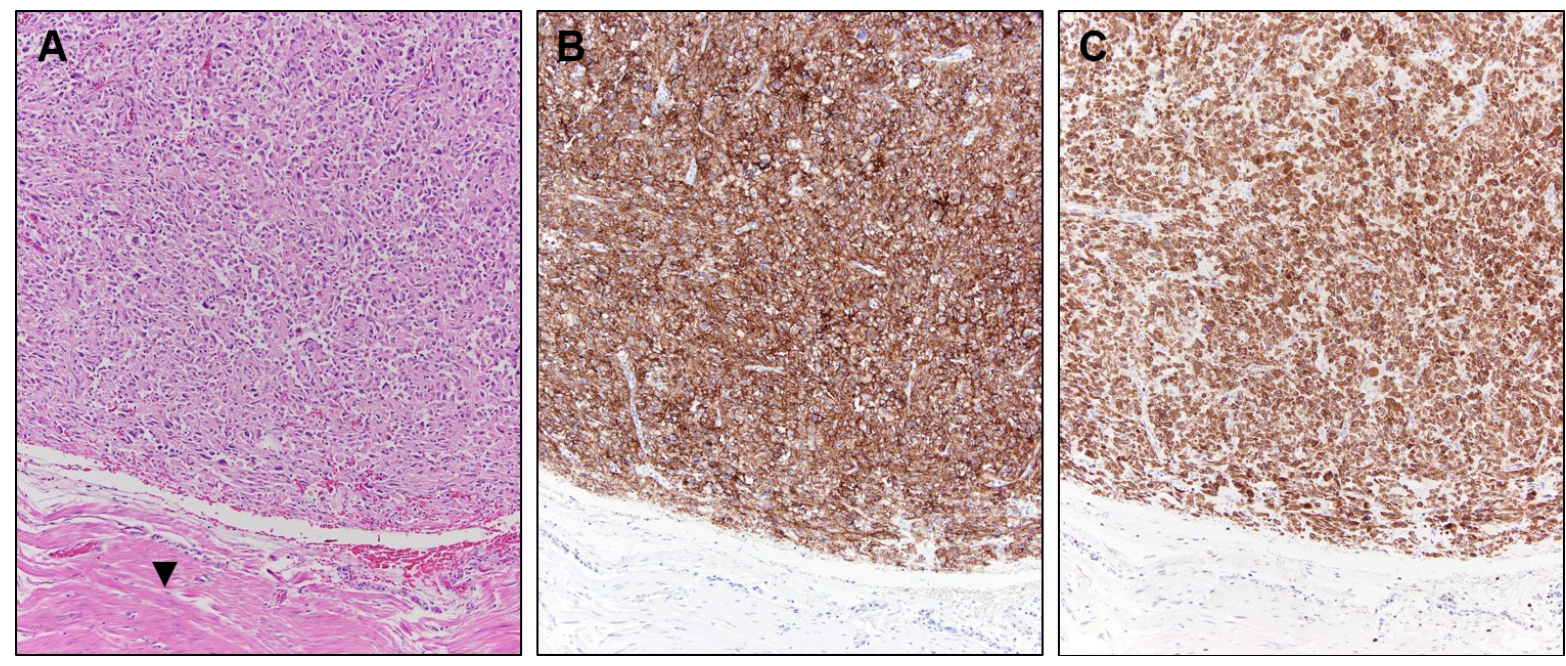

Figure 2 Case I GIST surgical resection pathology findings.

Notes: (A) Gastrointestinal stromal tumor involving the wall of the duodenum (arrowhead). The neoplastic cells showed epithelioid morphology with focal areas of spindle cell morphology. Mitotic figures were few ( $<5$ per 50 high-power fields). (H\&E stain; I00× original magnification) (B) DOGI immunohistochemistry showed diffuse positive staining. (C) Bcl-2 immunohistochemistry showed diffuse positive staining. (Immunohistochemistry with hematoxylin counterstain; I00× original magnification).

proceeded to pylorus-preserving pancreaticoduodenectomy, cholecystectomy, and retroperitoneal lymphadenectomy. Pathology revealed a $4.5 \mathrm{~cm}$ GIST invading the full thickness of duodenal wall, 1/18 lymph nodes involved by direct tumor extension, negative surgical margins, and less than five mitotic figures per 50 high-power field (HPF; Figure 2). No mutations in exons $9,11,13$, or 17 of the $c-K I T$ gene were reported. Immunohistochemical staining confirmed positivity of the GIST specimen for DOG1, a highly sensitive and specific marker for GIST, ${ }^{9}$ as well as diffuse strong positive staining for Bcl-2 (Figure 2).

Five months after pancreaticoduodenectomy, surveillance imaging revealed diffuse dissemination of disease. Tissue sampling was diagnostic of DLBCL with spread to both nodal and extranodal sites, including liver and bone. Owing to poor performance status, the patient pursued supportive care measures alone and passed away 3 months after relapse of lymphoma and 20 months after initial diagnosis of PBL.

\section{Case 2}

A 52-year-old woman presented with a screeningmammography-detected abnormality, diagnosed as an extranodal marginal zone (MALT) lymphoma of the right breast after excisional biopsy. This histologic diagnosis prompted definitive radiotherapy (RT) to the involved whole breast to $40 \mathrm{~Gy}$ in 20 daily fractions (representing the standard of care at the time; RT doses in this setting have since decreased). The patient's family history was notable for prostatic and lung adenocarcinomas in first-degree relatives, and her social history was negative for tobacco or alcohol usage.
Twelve months after completion of RT for her breast MALT lymphoma, follow-up surveillance PET/CT imaging revealed a new hypermetabolic large abdominopelvic mass as well as a new right orbital mass. Biopsy of the right orbital mass revealed recurrent extranodal marginal zone lymphoma, and for this she was treated with rituximab monotherapy for 9 months; this resulted in a metabolic complete response in the orbit and the patient has remained disease-free from her marginal zone lymphoma since that time ( 8 years ago) until her most recent follow-up. While the patient was receiving rituximab for her recurrent lymphoma, she underwent abdominopelvic mass excision which revealed a GIST of the small intestinal serosa measuring $21 \times 18 \mathrm{~cm}$ with four mitotic figures per $50 \mathrm{HPF}$, negative margins, and positive tumor staining CD117 and CD34 (Figure 3). She received adjuvant imatinib for 12 months. She went on to have four local (abdominopelvic) recurrences of her GIST, all managed with a combination of surgical resection and (neo)adjuvant imatinib over the subsequent 7 years to the time of last follow-up. Pathology from subsequent resections revealed exon $11 c$-KIT mutation. At the time of last follow-up, the patient was alive with recurrent disease (representing her fifth recurrence), with plans for neoadjuvant sorafenib and surgical resection.

\section{Discussion}

Here, we report on two cases of coincident PBL and GIST. From a statistical perspective, it is noteworthy that the chance of these entities occurring together randomly is $\sim 4.5$ per 100 billion if no interaction is assumed between 

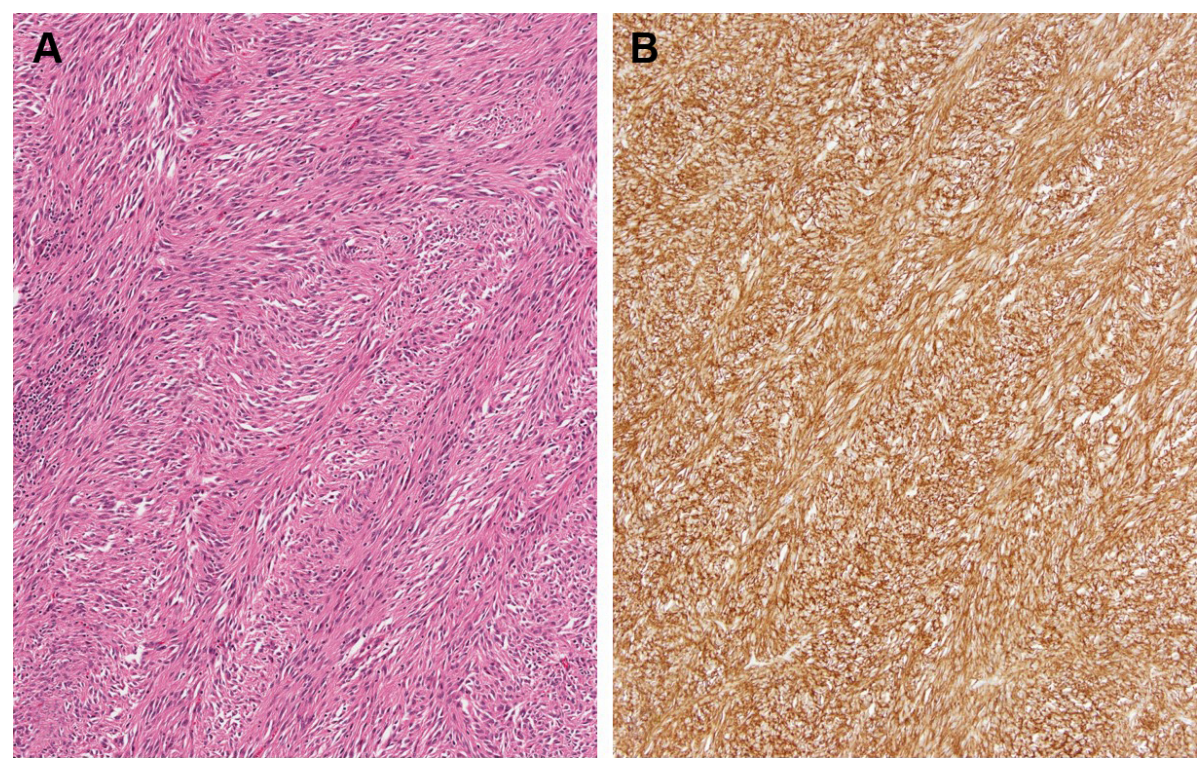

Figure 3 Case 2 GIST surgical resection pathology findings.

Notes: (A) GIST comprised of neoplastic cells with spindle cell morphology exhibiting a herringbone growth pattern. Mitotic figures were few (<5 per 50 high-power fields). (H\&E stain; 100× original magnification) (B) CDII7 immunohistochemistry showed diffuse positive staining. (Immunohistochemistry with hematoxylin counterstain; 100x original magnification).

Abbreviation: GIST, gastrointestinal stromal tumor.

these diagnoses. However, given the known association of GIST with NHL, it is more likely that as-of-yet unknown factors are driving the coincidence of GIST with PBL in these patients. ${ }^{10}$

According to one institutional review, patients with GIST and a second malignancy do not have significantly decreased survival. ${ }^{3}$ Moreover, the median OS for non-metastatic GIST in the imatinib era was 13.7 years in a study examining data from the Life Raft Group Registry, which parallels the prolonged survival despite numerous GIST relapses in the second case. ${ }^{11}$ These observations suggest that treatment strategies in cases of malignancies coinciding with GIST should often focus on the non-GIST malignancy.

Primary breast DLBCL represents a high-risk subtype of DLBCL, carrying substantial risk of central nervous system relapse as well as contralateral breast relapse. ${ }^{12,13}$ Data, including series from our institution, have demonstrated high rates of Bcl-2 overexpression in primary breast DLBCL, consistent with the patient from Case $1 .{ }^{14,15}$ Similarly, primary breast MALT lymphomas appear to carry higher risk of relapse, particularly distant relapse, compared with other primary MALT lymphomas, including those of the GI tract. ${ }^{16,17}$ Our data have shown overexpression of Bcl-10 in the vast majority of primary breast MALT lymphomas. ${ }^{15}$ Unfortunately, breast lymphoma tissue from the patient in Case 2 was not available for Bcl-10 testing, but institutional reports from other primary breast MALT lymphoma patients highlights characteristic Bcl-10 overexpression in these rare tumors. ${ }^{15}$ Both Bcl-2 and Bcl-10 appear to play critical roles in the pathogenesis of these respective lymphomas through anti-apoptotic activity. Bcl-2 in DLBCL has been shown to prevent chemotherapy-related apoptosis and, therefore, has been shown to carry prognostic significance. ${ }^{18,19} \mathrm{Bcl}-10$ in MALT lymphomas has similarly been shown to interact with the MALT1 gene product, resulting in inhibition of apoptosis. ${ }^{20}$ In both, primary breast DLBCL and MALT lymphoma, anti-apoptotic pathways appear to be central components of oncogenesis.

GISTs, mesenchymal tumors believed to arise from the interstitial cells of Cajal (part of the autonomic nervous system), are characterized by $c-K I T$ and PDGFR $\alpha$ mutations. ${ }^{21,22}$ While most GIST lesions have been shown to have $c$-KIT or PDGFR $\alpha$ mutations, $\sim 10 \%$ of these tumors are wild-type for both (the patient in Case 1 [Figure 2], whose GIST was negative for $c$-KIT mutations, though PDGFR $\alpha$ mutational testing was unavailable). ${ }^{23,24}$ It is noteworthy that exon 11 $c$-KIT mutant GIST patients have been recently shown to benefit from prolonged adjuvant imatinib for 3 years as opposed to 1 year. ${ }^{25}$ Consistent with these data, the patient in Case 2 experienced first relapse after only 1 year of adjuvant imatinib.

Looking toward common molecular oncogenic mechanisms between PBL and GIST, it has been shown that Bcl-2 is upregulated in GIST tissue as compared with normal 
gastric tissue. ${ }^{26}$ Survival advantages in PBLs through antiapoptotic activity of Bcl-2/Bcl-10, discussed previously, appear to potentially occur in GIST lesions as well. While Bcl-2 expression analysis was not available for GIST specimen for the patient in Case 2, the strongly positive Bcl-2 expression for the GIST specimen in Case 1 (Figure 2) supports concurrent overexpression of Bcl-2 in both this patient's GIST as well as her primary breast DLBCL. Common pathways promoting overexpression of anti-apoptotic proteins, such as Bcl-2, remain opaque. Preliminary data suggest that miR-21 may regulate Bcl-2 expression in both NHL and GIST cell lines, offering one hypothetical shared mechanism for upregulation of anti-apoptotic pathways across these different tumors. ${ }^{26-29}$ It is our hope that such speculation on common molecular pathways between these rare tumors spurs research into the underlying oncogenic pathways shared by these lesions, which may reveal as-ofyet-undiscovered oncogenic mechanisms in each of these tumors. With that in mind, the absence of $c$-KIT mutations in the GIST for Case 1 is noted, suggesting alternative molecular pathways that have not yet been characterized.

\section{Conclusion}

We report two patients with coinicident GIST and PBL. Given the improbability of stochastic co-occurrence of such uncommon tumors, there may a common underlying aberration (or aberrations) driving both lesions. In describing this association, we speculate that common anti-apoptotic pathways may be shared by these malignancies, and further research is needed to elucidate shared oncogenic mechanisms between GIST and PBL.

\section{Consent}

Written informed consent was obtained from the patients/ next of kin for this publication.

\section{Disclosure}

The authors report no conflicts of interest in this work.

\section{References}

1. Nilsson B, Bümming P, Meis-Kindblom JM, et al. Gastrointestinal stromal tumors: the incidence, prevalence, clinical course, and prognostication in the preimatinib mesylate era - a population-based study in western Sweden. Cancer. 2005;103(4):821-829.

2. Murphy JD, Ma GL, Baumgartner JM, et al. Increased risk of additional cancers among patients with gastrointestinal stromal tumors: a population-based study. Cancer. 2015;121(17):2960-2967.

3. Pandurengan RK, Dumont AG, Araujo DM, et al. Survival of patients with multiple primary malignancies: a study of 783 patients with gastrointestinal stromal tumor. Ann Oncol. 2010;21(10):2107-2111.
4. Agaimy A, Wünsch PH, Sobin LH, Lasota J, Miettinen M. Occurrence of other malignancies in patients with gastrointestinal stromal tumors. Semin Diagn Pathol. 2006;23(2):120-129.

5. Salar A, Ramón JM, Barranco C, et al. Double diagnosis in cancer patients and cutaneous reaction related to gemcitabine: Case 1. Synchronous mucosa-associated lymphoid tissue lymphoma and gastrointestinal stromal tumors of the stomach. J Clin Oncol. 2005;23(28): 7221-7223.

6. Kaffes A, Hughes L, Hollinshead J, Katelaris P. Synchronous primary adenocarcinoma, mucosa-associated lymphoid tissue lymphoma and a stromal tumor in a Helicobacter pylori-infected stomach. J Gastroenterol Hepatol. 2002;17(9):1033-1036.

7. Thomas A, Link BK, Altekruse S, Romitti PA, Schroeder MC. Primary breast lymphoma in the United States: 1975-2013. J Natl Cancer Inst. 2017;109(6)

8. Siddiqui FA, Maheshwari V, Alam K, Jain A. Coexistent non-Hodgkins lymphoma and ductal carcinoma breast: diagnosis on fine needle aspiration cytology. Diagn Cytopathol. 2011;39(10):767-769.

9. Hwang DG, Qian X, Hornick JL. DOG1 antibody is a highly sensitive and specific marker for gastrointestinal stromal tumors in cytology cell blocks. Am J Clin Pathol. 2011;135(3):448-453.

10. Karanikas M, Machairiotis N, Zarogoulidis P, et al. Non-Hodgkin lymphoma and GIST: molecular pathways and clinical expressions. Onco Targets Ther. 2012;5:433-438.

11. Call J, Walentas CD, Eickhoff JC, Scherzer N. Survival of gastrointestinal stromal tumor patients in the imatinib era: life raft group observational registry. BMC Cancer. 2012;12:90.

12. Aviv A, Tadmor T, Polliack A. Primary diffuse large B-cell lymphoma of the breast: looking at pathogenesis, clinical issues and therapeutic options. Ann Oncol. 2013;24(9):2236-2244.

13. Hosein PJ, Maragulia JC, Salzberg MP, et al. A multicentre study of primary breast diffuse large B-cell lymphoma in the rituximab era. Br J Haematol. 2014;165(3):358-363.

14. Yoshida S, Nakamura N, Sasaki Y, et al. Primary breast diffuse large B-cell lymphoma shows a non-germinal center B-cell phenotype. Mod Pathol. 2005;18(3):398-405.

15. Talwalkar SS, Valbuena JR, Abruzzo LV, et al. MALT1 gene rearrangements and NF-kappaB activation involving p65 and p50 are absent or rare in primary MALT lymphomas of the breast. Mod Pathol. 2006; 19(11):1402-1408.

16. Martinelli G, Ryan G, Seymour JF, et al. Primary follicular and marginal-zone lymphoma of the breast: clinical features, prognostic factors and outcome: a study by the International Extranodal Lymphoma Study Group. Ann Oncol. 2009;20(12):1993-1999.

17. Teckie S, Qi S, Lovie S, et al. Long-term outcomes and patterns of relapse of early-stage extranodal marginal zone lymphoma treated with radiation therapy with curative intent. Int J Radiat Oncol Biol Phys. 2015; 92(1):130-137.

18. Gascoyne RD, Adomat SA, Krajewski S, et al. Prognostic significance of Bcl-2 protein expression and Bcl-2 gene rearrangement in diffuse aggressive non-Hodgkin's lymphoma. Blood. 1997;90(1):244-251.

19. Miyashita T, Reed JC. Bcl-2 oncoprotein blocks chemotherapy-induced apoptosis in a human leukemia cell line. Blood. 1993;81(1):151-157.

20. Vega F, Medeiros LJ. Marginal-zone B-cell lymphoma of extranodal mucosa-associated lymphoid tissue type: molecular genetics provides new insights into pathogenesis. Adv Anat Pathol. 2001;8(6): 313-326.

21. Kindblom LG, Remotti HE, Aldenborg F, Meis-Kindblom JM. Gastrointestinal pacemaker cell tumor (GIPACT): gastrointestinal stromal tumors show phenotypic characteristics of the interstitial cells of Cajal. Am J Pathol. 1998;152(5):1259-1269.

22. Corless CL, Barnett CM, Heinrich MC. Gastrointestinal stromal tumours: origin and molecular oncology. Nat Rev Cancer. 2011;11(12): $865-878$.

23. Bai CG, Hou XW, Wang F, et al. Stem cell factor-mediated wild-type KIT receptor activation is critical for gastrointestinal stromal tumor cell growth. World J Gastroenterol. 2012;18(23):2929-2937. 
24. Lamba G, Ambrale S, Lee B, Gupta R, Rafiyath SM, Liu D. Recent advances and novel agents for gastrointestinal stromal tumor (GIST). J Hematol Oncol. 2012;5:21.

25. Joensuu H, Wardelmann E, Sihto H, et al. Effect of KIT and PDGFRA mutations on survival in patients with gastrointestinal stromal tumors treated with adjuvant imatinib: an exploratory analysis of a randomized clinical trial. JAMA Oncol. 2017;3(5):602-609.

26. Cao CL, Niu HJ, Kang SP, Cong CL, Kang SR. miRNA-21 sensitizes gastrointestinal stromal tumors (GISTs) cells to imatinib via targeting B-cell lymphoma 2 (Bcl-2). Eur Rev Med Pharmacol Sci. 2016; 20(17):3574-3581.
27. Go H, Jang JY, Kim PJ, et al. MicroRNA-21 plays an oncogenic role by targeting FOXO1 and activating the PI3K/AKT pathway in diffuse large B-cell lymphoma. Oncotarget. 2015;6(17):15035-15049.

28. Liu K, du J, Ruan L. MicroRNA-21 regulates the viability and apoptosis of diffuse large B-cell lymphoma cells by upregulating B cell lymphoma-2. Exp Ther Med. 2017;14(5):4489-4496.

29. Bai H, Wei J, Deng C, Yang X, Wang C, Xu R. MicroRNA-21 regulates the sensitivity of diffuse large B-cell lymphoma cells to the CHOP chemotherapy regimen. Int J Hematol. 2013;97(2):223-231.

\section{Publish your work in this journal}

OncoTargets and Therapy is an international, peer-reviewed, open access journal focusing on the pathological basis of all cancers, potential targets for therapy and treatment protocols employed to improve the management of cancer patients. The journal also focuses on the impact of management programs and new therapeutic agents and protocols on

\section{Dovepress}

patient perspectives such as quality of life, adherence and satisfaction. The manuscript management system is completely online and includes a very quick and fair peer-review system, which is all easy to use. Visit http://www.dovepress.com/testimonials.php to read real quotes from published authors. 